\title{
Sources of uncertainty in eddy covariance ozone flux measurements made by dry chemiluminescence fast response analysers
}

\author{
J. B. A. Muller ${ }^{1}$, C. J. Percival ${ }^{1}$, M. W. Gallagher ${ }^{1}$, D. Fowler ${ }^{2}$, M. Coyle ${ }^{2}$, and E. Nemitz \\ ${ }^{1}$ School of Earth, Atmospheric and Environmental Sciences, University of Manchester, Simon Building, Brunswick Street, \\ Manchester, M13 9PL, UK \\ ${ }^{2}$ Centre for Ecology \& Hydrology, Bush Estate, Penicuik, EH26 0QB, UK
}

Received: 11 August 2009 - Published in Atmos. Meas. Tech. Discuss.: 29 September 2009

Revised: 21 January 2010 - Accepted: 22 January 2010 - Published: 8 February 2010

\begin{abstract}
We present a systematic intercomparison study of eddy covariance ozone flux measurements made using two fast response dry chemiluminescence analysers. Ozone deposition was measured over a well characterised managed grassland near Edinburgh, Scotland, during August 2007. A data quality control procedure specific to these analysers is introduced. Absolute ozone fluxes were calculated based on the relative signals of the dry chemiluminescence analysers using three different methods and the results are compared for both analysers. It is shown that the error in the fitted analyser calibration parameters required for the flux calculations provides a substantial source of uncertainty in the fluxes. The choice of the calculation method itself can also constitute an uncertainty in the flux as the calculated fluxes by the three methods do not agree within error at all times. This finding highlights the need for a consistent and rigorous approach for comparable datasets, such as e.g. in flux networks. Ozone fluxes calculated by one of the methods were then used to compare the two analysers in more detail. This systematic analyser comparison reveals half-hourly flux values differing by up to a factor of two at times with the difference in mean hourly flux ranging from 0 to $23 \%$ with an error in the mean daily flux of $\pm 12 \%$. The comparison of analysers shows that the agreement in fluxes is excellent for some days but that there is an underlying uncertainty as a result of variable analyser performance and/or non-linear sensitivity.
\end{abstract}

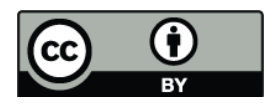

Correspondence to: J. B. A. Muller (jennifer.muller@postgrad.manchester.ac.uk)

\section{Introduction}

Tropospheric ozone $\left(\mathrm{O}_{3}\right)$ is an important greenhouse gas and thus influences climate, but it is also a secondary air pollutant relevant to air quality, with effects both on human health and vegetation. It is produced through photochemical reactions with precursor species such as nitrogen oxides and volatile organic compounds (VOCs). Precursor emissions have increased globally in the last few decades, resulting in a rise in ozone background concentrations (e.g. Derwent et al., 2007). These increased ground-level ozone background concentrations are of particular concern as ozone can cause adverse health effects in humans and vegetation (Ashmore, 2005; Fuhrer, 2009). To assess impacts on plants by ozone, a flux-based metric is being used for some vegetation risk assessments (Emberson et al., 2000). High quality ozone flux measurements are required to help validate the flux-based effects models. Ozone flux measurements and estimation of the deposition velocity/surface resistance are generally important for surface-atmosphere exchange modelling (Gruenhage et al., 2000). The work by Bassin et al. (2004) provides a recent example of the use of ozone flux measurements for model validation and development. Measurements also form the basis of parameterisations of deposition schemes in regional and global models, such as the EMEP model (Tuovinen et al., 2004). Similarly, the dry deposition sink provides one of the key uncertainties in the tropospheric ozone budget through its influence on ambient ozone concentrations. Hence uncertainties in the ozone deposition sink have an impact on and are reflected in the uncertainties in photochemical processes.

High quality ozone flux measurements are the only way to quantify fluxes directly and help understand destruction processes and relationships with other variables. The most direct method to measure fluxes is by eddy covariance. The

Published by Copernicus Publications on behalf of the European Geosciences Union. 
eddy covariance technique typically requires analysers that are fast response and sufficiently sensitive, although an alternative method of measuring ozone fluxes with a slowerresponse analyser has recently been suggested (Wohlfahrt et al., 2009), requiring large flux loss corrections which add uncertainty. A variety of suitable analysers exist for ozone, all of which are based on chemiluminescence. The reagents can be either in liquid, solid or gaseous form and hence the type of chemiluminescence is called wet, dry and gas-phase chemiluminescence respectively.

The use of the wet chemiluminescence method is not uncommon and it has advantages over the dry method (e.g. regarding signal drift and water vapour influence), but the operation can be hampered by liquid flow problems (e.g. Keronen et al., 2003) and the sensitivity can be lower than for the dry method. Gas phase chemiluminescence (GPC) is generally based on the homogeneous reaction with NO (Pearson, 1990) and used least frequently for surface flux measurements. This method is highly sensitive and reaction kinetics are better known than for any of the other chemiluminescence techniques. One of the main practical disadvantages of GPC is that NO is a toxic compound and is required as compressed gas during operation.

The dry chemiluminescence (DC) method has been used most regularly and successfully in a variety of environments and settings (e.g. Tuovinen et al., 1998, Bauer et al., 2000; Gallagher et al., 2001; Bassin, et al., 2004; Rummel et al., 2007). In DC air passes over a silica gel disc $(0.026 \mathrm{~m}$ in diameter) coated with the reagents gallic acid and coumarin 47 which produce photon emissions in the blue spectral range. The response time and sensitivity is flow rate dependent and high flow rates are used to operate in the flow-independent regime and achieve the fast response times required for eddy covariance measurements (Guesten et al., 1992). The chemiluminescence reaction with ozone consumes the reagent over time and discs need to be replaced periodically. Guesten et al. (1992) point out that the sensitivity of the discs depend on humidity and that sensitivity does not decrease linearly with time. Signal drift as caused by the consumption of the reactive compounds on the target disc can occur, which is one of the reasons why each disc has a limited lifetime and careful and frequent calibrations are required (Weinheimer, 2006). Knowledge about the relationship between sensitivity, temperature and humidity and its variability over time with increased accumulated ozone exposure is lacking and fundamental understanding about the reaction mechanism of chemiluminescence is also missing. Compared to the wet and gas-phase chemiluminescence techniques, this dry method is relatively low cost, low power and low weight and has become quite popular as operation of these systems is straightforward and simple.

Ozone fluxes have been measured by dry chemiluminescence for over 15 years using an analyser originally developed as an ozone sonde by GFAS ("Gesellschaft Für Angewandte Systemtechnik", Guesten et al., 1992), which has since then been reproduced by other groups (e.g. by NOAA-ATDD as detailed in Bauer et al., 2000). Fluctuations of ozone are detected optically via the fast heterogeneous chemiluminescence reaction of coumarin/gallic acid with ozone. The chemiluminescence target discs are made in a manner described by Speuser et al. (1989) and can be purchased from Bagus Consulting (Speyer, Germany). There is some variability in sensitivity as a result of the production process, storage and/or handling. Speuser et al. (1989) reported a variation in sensitivity of $\pm 5 \%$ of the pre-ozonised discs. The detection limit for ozone was found to be better than $50 \mathrm{ppt}$ of ozone (Guesten et al., 1992). Interferences in the chemiluminescence reaction have been tested by Guesten et al. $(1992,1995)$ who identified interference from water vapour and sulphur dioxide above $100 \mathrm{ppb}$, with response times of $22 \mathrm{~s}$ and $30 \mathrm{~min}$ respectively. No interference from NOx, PAN or $\mathrm{H}_{2} \mathrm{O}_{2}$ was observed.

In terms of the luminescence mechanism, the currently available literature contains a variety of ideas for the reactions of ozone with the reagents, but comprehensive laboratory studies would be required to provide more definitive answers. Hodgeson et al. (1970) suggested (for compound rhodamine B) the mechanism for chemiluminescence is based on a resonance energy transfer where the initial ozone during activation reacts with the dye to produce a dye molecule in an excited singlet state which then emits upon second exposure to ozone. This would be consistent with the reported loss of sensitivity from water vapour where the water vapour could quench the excited specie that is responsible for luminescence. Schurath et al. (1991) studied the dye coumarin 47 for chemiluminescence and found a response to ozone which showed great sensitivity when the disc was conditioned with ozonised air. They do not however provide a definitive answer to the question of the sensitizing mechanism. This conditioning or activation with ozonised air before use of the chemiluminescence discs has become standard practice and activation lengths in the range of 100 to $225 \mathrm{ppb} \mathrm{h}$ of ozone have been reported (McKendry et al., 1998; Schurath et al., 1991). Guesten et al. (1992) suggest that discs be replaced after $50 \mathrm{~h}$ of operation.

In this paper, ozone flux measurements by two effectively identical dry chemiluminescence instruments are presented and uncertainties in the fluxes examined with the help of the direct comparison of the analysers.

\section{Experimental}

\subsection{Measurement set up}

Continuous eddy covariance ozone flux measurements were made at a managed grassland site in Southern Scotland, UK, from 2 August to 3 September 2007. The Easter Bush site is located in the foothills of the Pentland Hills at $190 \mathrm{~m}$ above sea level and is surrounded by agricultural fields and farms 
(Milford et al., 2001). The fields surrounding the site are predominantly perennial rye-grass (Lolium perenne) and were grazed by sheep during August 2007. The canopy height reached about $0.1 \mathrm{~m}$. For the eddy covariance measurements a Metek USA-1 sonic anemometer (Meteorologische Messtechnik GmbH, Germany) was used for 3-D wind speed measurements at $2.5 \mathrm{~m}$ height. Fast ozone measurements were made using a GFAS ozone sonde OS-G-2 (Guesten et al., 1994) and a Rapid Ozone Flux Instrument (ROFI) which is in effect a clone of the GFAS ozone sonde built by CEH (Coyle, 2005). Both instruments were mounted on the eddy covariance mast and the sampling inlets placed alongside each other. Relative concentrations as $\mathrm{V}$ analogue signals from both analysers were sampled through the same anemometer interface box. A LabVIEW programme (National Instruments ${ }^{\mathrm{TM}}$ ) was used for data acquisition at a sampling frequency of $20 \mathrm{~Hz}$, online flux analysis and postsampling flux reanalysis. Absolute ozone concentration measurements from the Bush EMEP monitoring site situated about $400 \mathrm{~m} \mathrm{~S}$ of the Easter Bush flux site were used for the flux calculations. These concentration measurements were made using a TECO 49C UV ozone instrument (Thermo Electron Corp, USA) which is regularly calibrated to comply with the required monitoring network standards. Ozone concentrations were also measured at the Easter Bush site, but due to instrument failure data coverage for August 2007 was unsatisfactory for the eddy covariance flux calculations. The Bush ozone data were deemed acceptable as the regression between the Bush Monitoring site and the Easter Bush flux site for available ozone concentrations yielded a correlation coefficient $\left(R^{2}\right)$ of 0.95 with a slope of 1.05 and an intercept of $-3.23 \mathrm{ppb}$.

The chemiluminescence target discs for the GFAS and ROFI analysers were obtained from Bagus Consulting (Speyer, Germany) and changed in both instruments on 2, 6, 10, 14, 20, 24 and 28 August 2007. The disc surfaces were primed by pre-ozonisation at a nominal concentration of 100 ppb using a GFAS ozoniser unit for 90 min immediately before exchanging the disc in the analysers.

\subsection{Analysis}

As the dry chemiluminescence method provides a relative measure of ozone, absolute ozone concentrations, measured generally by a UV absorption instrument, are needed to calibrate the relative ozone signal. There are different approaches to obtaining absolute ozone fluxes from the relative signal and most authors do not clearly report what method is used. Since there has been no systematic investigation into the calculation methods, three different approaches have been used and compared here.

\subsubsection{Eddy covariance method}

Eddy covariance allows the direct measurement of the vertical flux at a single height. Any scalar, such as e.g. the instantaneous vertical wind component $(w)$ can be Reynoldsdecomposed into the time-averaged component $(\bar{w})$ and deviating or fluctuating part $\left(w^{\prime}\right)$ :

$w=\bar{w}+w^{\prime}$

The mean vertical turbulent flux $\left(F_{\chi}\right)$ is given by the covariance of the fluctuating part of the vertical wind component $\left(w^{\prime}\right)$ and the fluctuating part of the scalar tracer $\left(\chi^{\prime}\right)$, averaged over a given period of time, e.g. $30 \mathrm{~min}$ :

$F_{\chi}=\overline{w^{\prime} \chi^{\prime}}$

The ozone chemiluminescence analysers output the signal in units of Volts $(\mathrm{V})$ and the fluctuating part of the relative ozone concentration $\left(X^{\prime}\right)$ is used to calculate a relative ozone flux $\left(\overline{w^{\prime} X^{\prime}}\right.$, with units of $\left.\mathrm{V} \mathrm{m} \mathrm{s}^{-1}\right)$. The mean relative ozone concentration $(X$, units of $\mathrm{V})$ is also calculated, as well as the mean absolute ozone concentration $\left(\chi_{03} \mu \mathrm{g} \mathrm{m}^{-3}\right)$ which is used for calibration purposes. (Please note, no overbar is used for these time-averaged concentrations in order to keep later equations simple.)

\subsubsection{Spectral analysis}

The power and co-spectra are calculated by forward Fourier transforms. This converts from physical space to frequency space which allows probing the contribution to the total variance of a scalar at particular frequencies. The discrete power spectral density is defined as

$S_{A}(f)=2 \cdot\left|F_{A}(f)\right|^{2} / \sigma_{\text {total }}^{2}$

where $S_{A}$ is the discrete power spectral density of time series $A, f$ is the natural frequency $(\mathrm{Hz}),\left|F_{A}(f)\right|^{2}$ are the summed Fourier transform coefficients of both imaginary and real parts of time series $A$ and $\sigma_{\text {total }}^{2}$ is the total variance of $A$ (and sum of the spectral energies) (Stull, 1988). Here the power spectral analysis is performed for the relative ozone signal.

The co-spectrum $\left(\mathrm{Co}_{\mathrm{AB}}\right)$ is calculated by the sum of products of real and imaginary parts of the Fourier transform of time series A and B (Stull, 1988) and the co-spectral density (Co) is defined here as

$\mathrm{Co}=\mathrm{Co}_{\mathrm{AB}}(f) / \sigma_{(\mathrm{AB})}$

where $\sigma_{(\mathrm{AB})}$ is the co-variance of $\mathrm{A}$ and $\mathrm{B}$.

The co-spectral coefficients can take positive or negative values. The negative values have been inverted to positive values to allow plotting on a log scale.

Both power spectra and co-spectra are weighted by the natural frequency $f$, and plotted against the normalised frequency $n$, which allows the comparison of spectra calculated 
for different meteorological conditions and measured at different heights. The natural frequency $n$ is defined as

$n=f \cdot z / U$

where $z$ is the measurement height above the surface and $U$ is the mean horizontal windspeed.

\subsubsection{Eddy covariance data processing}

The high frequency eddy covariance data series were despiked, but not de-trended and wind vectors were rotated according to the planar-fit method (Wilczak et al., 2001). The raw ozone time series was shifted according to the inlet lag time, based on the maximum correlation of vertical velocity $w^{\prime}$ and raw ozone signal $X^{\prime}$. Fluxes were averaged over $30 \mathrm{~min}$ and filtered for stationarity according to Foken and Wichura (1996). Corrections for density fluctuations due to temperature and water vapour were applied (Lee et al., 2004). No further flux loss corrections were made. Data were also filtered for obstructed wind direction and for very low friction velocity, $u^{*}$, values below $0.03 \mathrm{~m} \mathrm{~s}^{-1}$.

Errors or uncertainties by the eddy covariance method have not been discussed in detail here and a full treatment of errors from eddy covariance in general can be found for instance by Businger (1986) and Finkelstein and Sims (2001).

\subsubsection{Absolute ozone fluxes by Ratio Method}

The Ratio Method (RM) does not require the calculation of a calibration factor obtained by the relative ozone fluctuation measurements and absolute ozone concentrations. Instead, only average ozone concentrations at the frequency of the flux averaging period are needed to obtain absolute fluxes.

The ozone deposition velocity $v_{d}$ (in units of $\mathrm{m} \mathrm{s}^{-1}$ ) can be calculated by the relative ozone flux $\left(\overline{w^{\prime} X^{\prime}}\right.$ in units of $\mathrm{V} \mathrm{m} \mathrm{s}^{-1}$ ) divided by the mean relative ozone concentration $(X$, in units of $\mathrm{V})$ :

$v_{d}=-\frac{\overline{w^{\prime} X^{\prime}}}{X}$

To obtain the absolute flux of ozone $\left(F_{O 3}\right.$ in $\left.\mu \mathrm{g} \mathrm{m}^{-2} \mathrm{~s}^{-1}\right)$, the deposition velocity is multiplied by the mean absolute ozone concentration $\left(\chi_{03} \mu \mathrm{g} \mathrm{m}^{-3}\right)$ :

$F_{O 3}=-v_{d} \cdot \chi_{O 3}$

In this study, fluxes were calculated over 30 min periods, so the absolute flux can be described by:

$F_{O 3(30)}=\frac{\overline{w^{\prime} X^{\prime}}(30)}{X_{(30)}} \cdot \chi_{O 3(30)}$

One implied assumption in this method is that the analyser output is proportional to $\chi_{O 3}$ with a zero offset. If that is not the case, the absolute flux will be overestimated for negative offset values and underestimated for positive offset values.

\subsubsection{Absolute ozone fluxes by Ratio Offset Method}

The Ratio Offset Method (ROM) is based on the Ratio Method (Eq. 8) with the modification that accounts for the offset in the analyser. The offset value is effectively the mean output at zero ozone concentration, as obtained by a regression of the mean output, $X$, against absolute ozone concentrations, $\chi_{03}$. As the offset might change on a disc by disc basis, an offset value is obtained for each disc period by a least squares fit using $15 \mathrm{~min}$ averaged data. (15 min averages resulted in higher regression coefficients than the use of $30 \mathrm{~min}$ data). For the number of values from $i=1$ to $\mathrm{N}$ in each disc period, the offset $(a)$ can be estimated as

$a=\frac{\Sigma X_{(15) i} \cdot \Sigma \chi_{O 3(15) i}^{2}-\Sigma \chi_{O 3(15) i} \cdot X_{(15) i} \cdot \Sigma \chi_{O 3(15) i}}{\mathrm{~N} \cdot \Sigma \chi_{O 3(15) i}^{2}-\left(\Sigma \chi_{O 3(15) i}\right)^{2}}$

Subtracting the offset from the mean output, Eq. 8) is modified to obtain absolute half-hourly flux as follows

$$
=\frac{F_{O 3(30)}}{X_{(30)}-\frac{\Sigma X_{(15) i} \cdot \Sigma \chi_{O 3(15) i}^{2}-\Sigma \chi_{O 3(15) i} \cdot X_{(15) i} \cdot \Sigma \chi_{O 3(15) i}}{\mathrm{~N} \cdot \Sigma \chi_{O 3(15) i}^{2}-\left(\Sigma \chi_{O 3(15) i}\right)^{2}}} \cdot \chi_{O 3(30)}
$$

The error estimates in $\mathrm{F}_{O 3}$ as calculated by ROM are estimated using two times the standard deviation, i.e. $2 \sigma$, in the offset value a obtained by the regression of raw ozone signal with absolute ozone concentrations (Eq. 9).

\subsubsection{Absolute ozone fluxes by Disc Calibration Method}

The Disc Calibration Method (DCM) applies a calibration factor to the raw flux $\left(\mathrm{V} \mathrm{m} \mathrm{s}^{-1}\right)$ for each disc period. The calibration factor is obtained by regressing the absolute ozone concentration measurements, $\chi_{O 3}$, against the averaged analyser output $(X)$. A linear fit using the least squares method is used assuming that the sensitivity is linear across the range of ozone concentrations and linear with time. The calibration factor and associated error depend on how well the voltage output correlates with absolute ozone concentration, and the choice of averaging time can make a difference. As for the offset (see above), calibration factors have been calculated using $15 \mathrm{~min}$ averaged data, as better correlations were achieved with 15 than 30 min data. The least square method allows estimation of the slope of the fit $(c)$, for each disc period with data $\left(X, \chi_{O 3}\right)$ values ranging from $i=1$ to $\mathrm{N}$, viz

$c=\frac{\mathrm{N} \cdot \Sigma X_{(15) i} \cdot \chi_{O 3(15) i}-\Sigma X_{(15) i} \cdot \Sigma \chi_{O 3(15) i}}{\mathrm{~N} \cdot \Sigma X_{(15) i}^{2}-\left(\Sigma X_{(15) i}\right)^{2}}$

The slope of the fit or calibration factor $c,\left(\mu \mathrm{g} \mathrm{m}^{-3} \mathrm{~V}^{-1}\right)$ is multiplied with the raw flux $\left(\overline{w^{\prime} X^{\prime}}\right.$, with units of $\left.\mathrm{V} \mathrm{m} \mathrm{s}^{-1}\right)$ to obtain absolute ozone fluxes:

$F_{O 3(30)}=c \cdot \overline{w^{\prime} X^{\prime}}(30)$ 


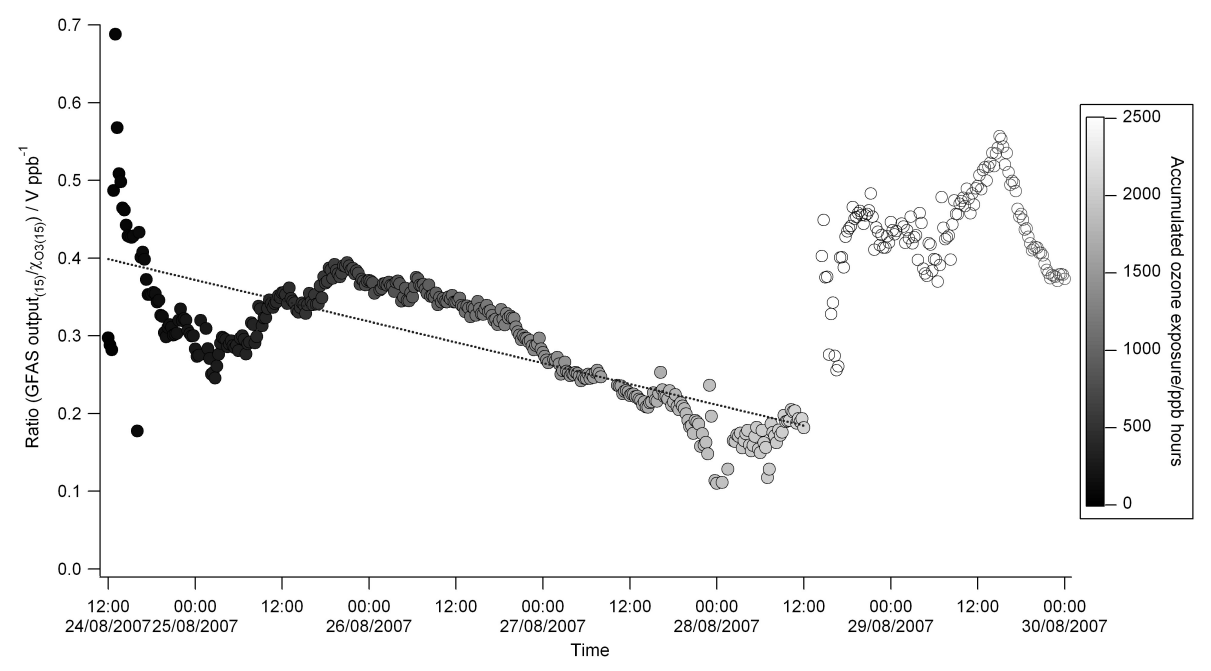

Fig. 1. Ratio of 15 min relative ozone concentration (GFAS mean output) and absolute ozone concentration for Disc Period 6 (closed symbols) and Disc Period 7 (open symbols). Fitted slope, i.e. trend in ratio is $-6.21 \times 10^{-7} \mathrm{~V} \mathrm{ppb}^{-1}$.

The absolute flux can thus be described for each disc period separately as

$$
F_{O 3(30)}=\overline{w^{\prime} X^{\prime}}(30) \cdot \frac{\mathrm{N} \cdot \Sigma X_{(15) i} \cdot \chi_{O 3(15) i}-\Sigma X_{(15) i} \cdot \Sigma \chi_{O 3(15) i}}{\mathrm{~N} \cdot \Sigma X_{(15) i}^{2}-\left(\Sigma X_{(15) i}\right)^{2}}
$$

Error estimates for the flux values from DCM are calculated by using two times the standard deviation in the estimated calibration factor $c$.

The Disc Calibration Method has an implicit assumption that there is no degradation of sensitivity over the time period considered. This should be a reasonable assumption provided that the discs are changed before such degradation occurs. Here, only the slope of the regression (i.e. the calibration factor $c$ ) and not an intercept is used, as in this approach the constant intercept would not correlate with $w^{\prime}$ (which is zero on average) and thus the intercept would not contribute to the flux.

\subsection{Data quality control procedure}

To ensure that the data quality from the chemiluminescence analysers is satisfactory, the following quality control (QC) procedure has been implemented to identify and discard erroneous or low quality data points:

1. Discard data points during logged downtime, power cuts etc.

2. As the first few hours of a disc period can contain spurious values, all data up to $2 \mathrm{~h}$ immediately after renewing the chemiluminescence target disc are deleted.

3. By visual inspection, compare time series $(15 \mathrm{~min})$ of the average ozone signal $(X)$ and $\chi_{03}$ to find and remove outliers or periods of erroneous data points.
4. Plot $15 \mathrm{~min}$ (or other higher resolution than flux average period) $\chi_{03}$ vs average output $X$ (voltage) on a disc by disc basis, colour coding with disc hours. Discard outliers and potentially clusters/periods of questionable data, especially occurring towards the end of a disc period, when disc sensitivity might be reduced. When a reduction in sensitivity occurs, the ratio between the relative voltage output and absolute ozone concentration is decreased. Figure 1 shows data from a period where a loss in sensitivity of the chemiluminescence target disc occurred with time (Disc Period 6 - closed symbols, Disc Period 7 - open symbols) and more variability occurred towards the end of the disc period. The step change in the ratio from Disc Period 6 to 7 also illustrates that the sensitivity can change from one disc to another.

5. Obtain calibration factors, i.e. slopes of a linear fit between $\chi_{O 3}$ and voltage output $X$, and $R^{2}$ of the fit. If the $R^{2}$ value is above 0.5 , the data quality is acceptable and the calibration factor can be applied to the raw voltage flux. If the $R^{2}$ value is below 0.5 , it might be possible to remove further outliers/data values at the end of the disc period to improve the correlation. If this is not successful, the whole period has to be identified as not reliable and data points need to be discarded. Use the same dataset for obtaining the offset for ROM.

6. A final visual inspection for outliers is recommended on the absolute flux data as not all erroneous flux values correspond to erroneous average voltage output and are thus removed by the above steps.

This procedure is recommended even when the RM and ROM are used because questionable discs and periods as well as individual outliers are removed with this protocol. 
Table 1. Statistics based on whole GFAS and ROFI flux datasets for all calculation methods, using paired samples only. All values are in units of $\operatorname{ng~m}^{-2} \mathrm{~s}^{-1}$.

\begin{tabular}{llccccccc}
\hline & & Min. & Lower Quart. & Median & Upper Quart. & Max. & Mean & $2 \sigma$ mean error \\
\hline \multirow{2}{*}{ RM } & GFAS & -914 & -303 & -195 & -129 & 159 & -218 & n/a \\
& ROFI & -753 & -220 & -138 & -87 & 105 & -161 & n/a \\
\multirow{2}{*}{ ROM } & GFAS & -928 & -250 & -158 & -104 & 45 & -180 & \pm 26 \\
& ROFI & -632 & -230 & -147 & -95 & 114 & -170 & \pm 33 \\
\multirow{2}{*}{ DCM } & GFAS & -420 & -175 & -108 & -69 & 49 & -125 & \pm 17 \\
& ROFI & -453 & -147 & -101 & -68 & 45 & -112 & \pm 16 \\
\hline
\end{tabular}

\subsection{Meteorological conditions and analyser performance}

The UK national weather report (Weather, 2007) described August 2007 as a "quiet, rather cool month" and Scotland was mainly under the influence of low pressure systems, including fronts passing over the experimental site during the first three weeks. During the last two weeks of August, high pressure systems and anticyclonic circulation produced somewhat warmer temperatures and light winds. Overall, conditions were typical for the site with an average windspeed (at $2.5 \mathrm{~m}$ ) of $3.35 \mathrm{~m} \mathrm{~s}^{-1}$ (range from 0.06 to $8.1 \mathrm{~m} \mathrm{~s}^{-1}$ ), an average temperature of $10.9^{\circ} \mathrm{C}$ (range from 5.1 to $21.4{ }^{\circ} \mathrm{C}$ ) and an average relative humidity of $80.8 \%$ (range from 42.1 to $100 \%$ ). The mean ozone concentration of $24.2 \mathrm{ppb}$, ranging from 4.0 to $57.8 \mathrm{ppb}$ of ozone, is typical for the site and time of the year.

Data capture for absolute ozone concentrations from the UV absorption instrument was good with only $6 \%$ data loss as a result of a daily calibration routine and additional short downtime. For the eddy covariance measurements, the dry chemiluminescence analysers were running for 1506 halfhourly periods. After full quality control of the flux data, the final data capture was $75 \%$ for the GFAS and $62 \%$ for the ROFI analyser. The loss of data is attributed to the following effects: $2 \%$ of the data was discarded because of the removal of two hours at the beginning of each disc period, $2 \%$ as a result of the wind direction filter, $2 \%$ because of the low $u^{*}$ filter, 7 and $8 \%$ for GFAS and ROFI respectively for conditions of non-stationarity and 12 and $25 \%$ for GFAS and ROFI respectively for downtime/outliers/end-of-disc period sensitivity loss (details see quality control procedure above).

\section{Results and Discussion}

\subsection{Comparison of the calculation methods and resulting uncertainties}

The three different methods (RM, ROM and DCM) were used to calculate absolute ozone fluxes and Fig. 2 shows the time series for the ROFI and GFAS analyser for each method.
The comparison of the calculation methods for the whole dataset shows that the DCM produces smaller mean and median deposition fluxes than the RM and ROM (Table 1), with a smaller range of 30-min values. There is no obvious systematic difference between the RM and ROM for both analysers. The overall statistics can only reveal limited information about the data and it is evident from the time series (Fig. 2) that there are some periods when the agreement of the different methods is better (e.g. 15 August 2007) than at other times (e.g. 10 August 2007).

Table 2 shows the basic statistics for each method and each analyser for each disc period. The median and mean deposition fluxes in each disc period are smallest for the DCM. In case of the GFAS, the ROM generally gives values that are in closer agreement with the DCM than the RM. This is not the case for the ROFI where there is no such systematic difference between the RM and ROM. This would imply that the offsets on the GFAS are larger than on the ROFI which is indeed the case: Based on the absolute values of the offsets, the mean offset for the GFAS is $2.0 \mathrm{~V}$ and $1.1 \mathrm{~V}$ for the ROFI. For the GFAS 6 out of 7 offsets were negative whereas for the ROFI 2 out of 7 were negative. ROM should provide more accurate flux estimates than RM when the offset is nonzero and changes from disc to disc period as is the case for the ROFI analyser, as illustrated for disc periods 1 and 4 in Fig. 3.

The comparison of methods shows there can be differences in measured ozone fluxes depending on the choice of calibration or calculation method. All three methods presented here are in principle viable options to calculate ozone fluxes, although it could be argued that the offsets in this study are large enough to preferably choose the DCM or ROM over the RM. The observed differences between the methods add to the uncertainty of the calculated flux and for a substantial part, the difference cannot be explained by the error estimate associated with the calibration fit as illustrated in Fig. 2. This naturally raises the question as to which method gives values closest to the true flux values and whether the assumptions the methods are based on are fulfilled. One of the main differences in the methods is the use of a fitted curve obtained using the whole disc period 15 min data in the DCM 


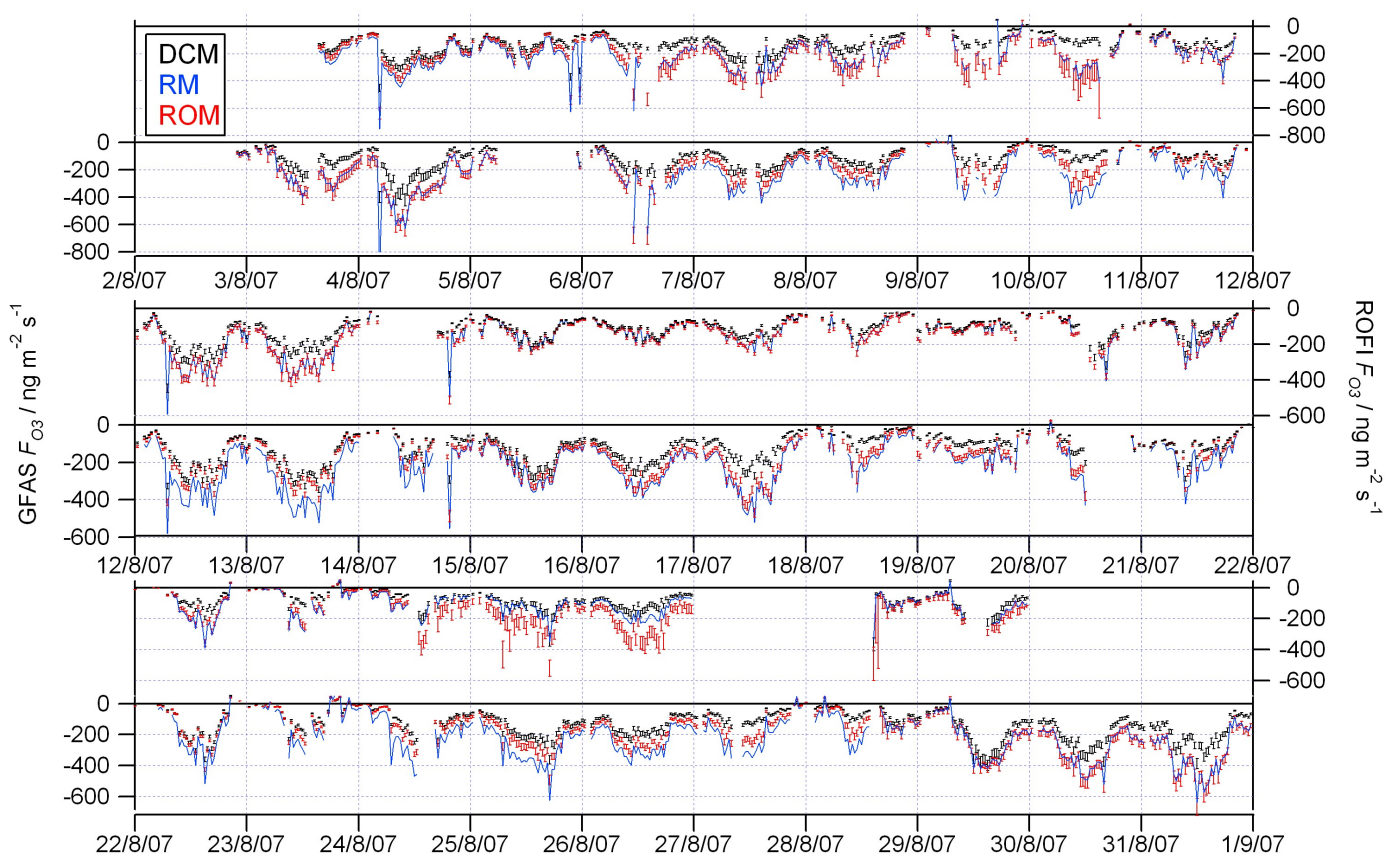

Fig. 2. Time series of ROFI and GFAS fluxes for each calculation method, with error bars based on $2 \mathrm{x}$ standard deviation in the parameter fit of the calculation method. Top two panels cover the period 2 August 2007 to 12 August 2007, middle two panels cover the period from 12 August 2007 to 22 August 2007 and the bottom two panels show period 22 August 2007 to 1 September 2007.

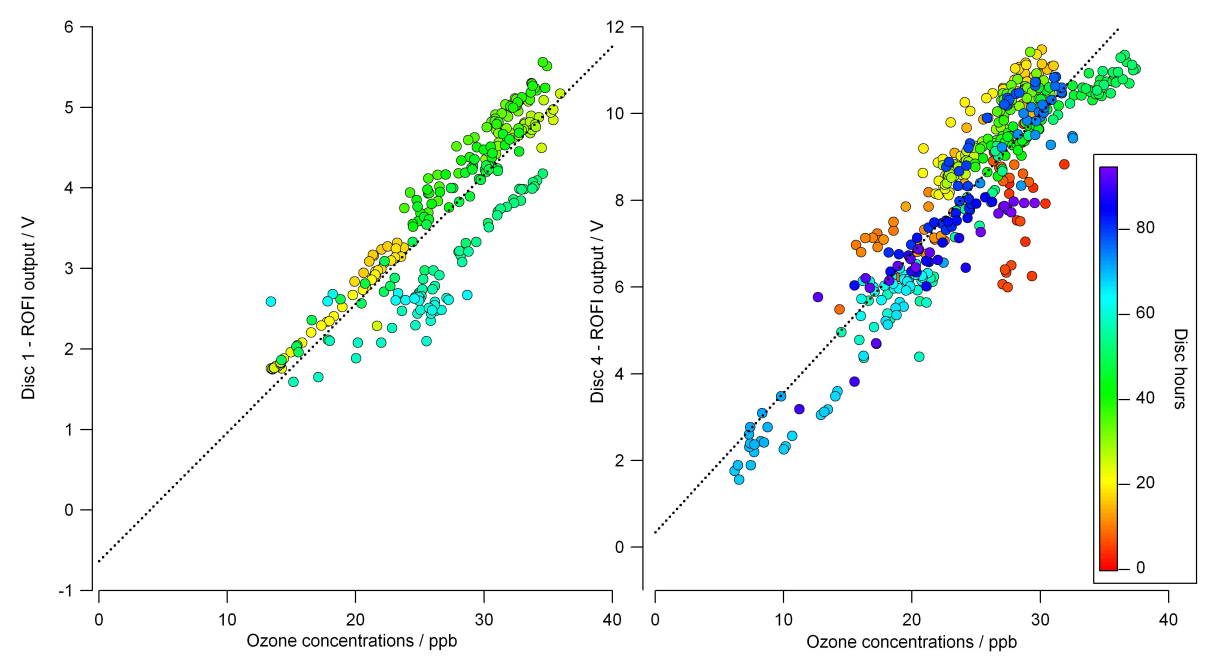

Fig. 3. Offsets in analyser output vary from disc to disc. Negative offset of $-0.65 \mathrm{~V}$ for disc period 1 (left panel) and positive offset of $0.35 \mathrm{~V}$ for disc period 4 (right panel). The colour of the symbols is based on the number of $h$ the disc has been exposed to the atmosphere.

and ROM. In comparison RM uses only single 30 min average values. All the methods rely on the assumption that the fast fluctuations remain proportional to the mean output either for the entire disc period (DCM and ROM) or for the flux averaging period (RM). The fulfilment of the proportionality assumption for RM for each flux averaging period could be tested using high resolution absolute ozone concentrations (tens of seconds to $1 \mathrm{~min}$ averages), provided the range of concentrations in the flux-averaging period is sufficient for a reliable regression. These high resolution absolute ozone data were not available for this study, so the proportionality test (regression) for the entire disc period was used here. From the regression plots it is sometimes visible that sensitivity decreases with time and can include fast changes, as exemplified by Fig. 1, and an average calibration factor will give slightly overestimated fluxes in the beginning and underestimated fluxes at the end of the disc period. The effect is estimated to be small, but for some disc periods it is a 
Table 2. Basic statistics for each disc period for all methods RM, ROM, DCM for both GFAS and ROFI analysers, using paired samples only. Median values in bold and mean values in italic. $2 \sigma$ error is the mean error for that disc period based on the standard deviation in the regression parameter fit. All values are in units of $\mathrm{ng} \mathrm{m}^{-2} \mathrm{~s}^{-1}$

\begin{tabular}{|c|c|c|c|c|c|c|c|c|c|c|c|c|c|c|}
\hline & \multicolumn{2}{|c|}{ Disc Period 1} & \multicolumn{2}{|c|}{ Disc Period 2} & \multicolumn{2}{|c|}{ Disc Period 3} & \multicolumn{2}{|c|}{ Disc Period 4} & \multicolumn{2}{|c|}{ Disc Period 5} & \multicolumn{2}{|c|}{ Disc Period 6} & \multicolumn{2}{|c|}{ Disc Period 7} \\
\hline & GFAS & ROFI & GFAS & ROFI & GFAS & ROFI & GFAS & ROFI & GFAS & ROFI & GFAS & ROFI & GFAS & ROFI \\
\hline \multicolumn{15}{|c|}{ RATIO METHOD (RM) } \\
\hline Minimum & -914 & -753 & -486 & -440 & -582 & -593 & -553 & -500 & -516 & -390 & -624 & -349 & -417 & -258 \\
\hline Lower Quart. & -337 & -297 & -314 & -287 & 335 & -293 & -273 & -155 & -248 & -178 & 3 & -166 & -206 & -138 \\
\hline Median & -232 & -225 & -233 & -209 & -213 & -204 & -172 & -115 & -143 & -101 & -247 & -108 & -148 & -97 \\
\hline Upper Quart. & -140 & -130 & -135 & -139 & -124 & -116 & -135 & -80 & -85 & -57 & -165 & -77 & -99 & -69 \\
\hline Maximum & -39 & -58 & 159 & & 15 & 14 & -31 & -21 & 67 & 56 & -87 & -35 & 35 & 40 \\
\hline Mean & -264 & -234 & -225 & -210 & -237 & -212 & -202 & -120 & -167 & -118 & -256 & -122 & -165 & -108 \\
\hline $2 \sigma$ error & $\mathrm{n} / \mathrm{a}$ & $\mathrm{n} / \mathrm{a}$ & $\mathrm{n} / \mathrm{a}$ & $\mathrm{n} / \mathrm{a}$ & $\mathrm{n} / \mathrm{a}$ & $\mathrm{n} / \mathrm{a}$ & $\mathrm{n} / \mathrm{a}$ & $\mathrm{n} / \mathrm{a}$ & $\mathrm{n} / \mathrm{a}$ & $\mathrm{n} / \mathrm{a}$ & $\mathrm{n} / \mathrm{a}$ & $\mathrm{n} / \mathrm{a}$ & $\mathrm{n} / \mathrm{a}$ & $\mathrm{n} / \mathrm{a}$ \\
\hline \multicolumn{15}{|c|}{ RATIO OFFSET METHOD (ROM) } \\
\hline Minimum & -928 & -632 & -362 & -482 & -415 & -604 & -486 & -510 & -427 & -357 & -492 & -516 & -425 & -289 \\
\hline Lower Quart. & -341 & -254 & -238 & -309 & -251 & -299 & -244 & -157 & -182 & -161 & -269 & -259 & -213 & -162 \\
\hline Median & -235 & -194 & -175 & -228 & -213 & -204 & -151 & -117 & -114 & -89 & -185 & -178 & -153 & -118 \\
\hline Upper Quart & -144 & -112 & -102 & -148 & -84 & -119 & -118 & -82 & -49 & -42 & -123 & -132 & -104 & -83 \\
\hline Maximum & -39 & -46 & 61 & 114 & 9 & 15 & -18 & -22 & 45 & . & - & -80 & 38 & 55 \\
\hline Mean & -268 & -198 & -170 & -229 & -170 & -216 & -177 & -123 & -124 & -106 & -198 & -204 & -171 & -130 \\
\hline $2 \sigma$ error & \pm 57 & \pm 26 & \pm 31 & \pm 77 & \pm 14 & +26 & +22 & \pm 10 & \pm 10 & \pm 16 & \pm 26 & \pm 60 & \pm 33 & \pm 31 \\
\hline \multicolumn{15}{|c|}{ DISC CALIBRATION METHOD (DCM) } \\
\hline Minimum & -420 & -453 & -307 & -458 & -347 & -447 & -292 & -368 & -360 & -258 & -365 & -337 & -399 & -227 \\
\hline Lower Quart. & -209 & -199 & -156 & -154 & -220 & -217 & -166 & -128 & -153 & -100 & -197 & -129 & -149 & -129 \\
\hline Median & -130 & -143 & -100 & -114 & -126 & -149 & -99 & -96 & -102 & -67 & -142 & -99 & -113 & -90 \\
\hline Upper Quart & -68 & -93 & -63 & -82 & -71 & -90 & -73 & -68 & -38 & -27 & -94 & -70 & -65 & -62 \\
\hline Maximum & -24 & -31 & 46 & 45 & 7 & 9 & -10 & -13 & 49 & 33 & -42 & -33 & 27 & 43 \\
\hline Mean & -150 & -153 & -107 & -121 & -147 & -153 & -119 & -100 & -106 & -69 & -149 & -103 & -127 & -98 \\
\hline $2 \sigma$ error & \pm 31 & \pm 19 & \pm 18 & \pm 23 & \pm 13 & \pm 16 & \pm 15 & \pm 8 & \pm 9 & \pm 9 & \pm 22 & \pm 26 & \pm 21 & \pm 23 \\
\hline
\end{tabular}

potential source of deviation from the true flux value. This would affect DCM and ROM, but not RM. However to be confident that RM gives a correct flux value, the proportionality of raw output and absolute ozone concentrations would have to be tested for each flux averaging period. It is possible to minimise such errors in DCM and ROM by choosing shorter periods over which to calculate calibration factors, however there is a trade-off with potentially larger errors in the fitted parameters with fewer points used in the regression calculation.

Each method is based on the basic principle that the mean output remains linearly proportional to the fast fluctuations over time and the whole range of ozone concentrations. This assumption of the mean output being proportional to the fast fluctuations and how that relationship might change with accumulated ozone exposure can only be comprehensively investigated in controlled laboratory studies, which is beyond the scope of this study. The data filter also requires the setting of a threshold for the required goodness of the fit that is acceptable. In this study the $R^{2}$ threshold chosen was 0.5 and for most discs this is an achievable value. However there are cases when the scatter can be considerable and a $R^{2}$ value of 0.5 is not achieved before removing data points at the end of the disc periods on the grounds of reduction in sensitiv- ity. This shows that there can be periods when the assumption of linearity fails. If RM is used without the proposed data filter, these periods of non-linearity are not identified and in the absence of a non-linearity test for every flux averaging period, the approach used here gives confidence in the accuracy of the flux data from this method. Another inherent problem with $\mathrm{RM}$ is that it assumes that there is no voltage offset. In fact the offset values (as used in ROM) in this study were non-zero, both positive and negative, ranging from -4.2 to $3.2 \mathrm{~V}$. To investigate whether the offset affects calculated fluxes more when the output voltage is low, the absolute difference in flux between the methods was regressed against the mean voltage output. The largest $R^{2}$ value was 0.1 which indicates that no correlation between the spread of values and mean output voltage exists and that the offset is somehow dependent on voltage output.

In this study, ROM could be considered preferable to RM as the offsets in the analysers were non-zero and varied from disc to disc. Although ROM fluxes on the whole agree more closely with DCM fluxes, the ROM and DCM flux values are still not generally within the error estimates as obtained by the uncertainty in the regression parameters (Fig. 2). 


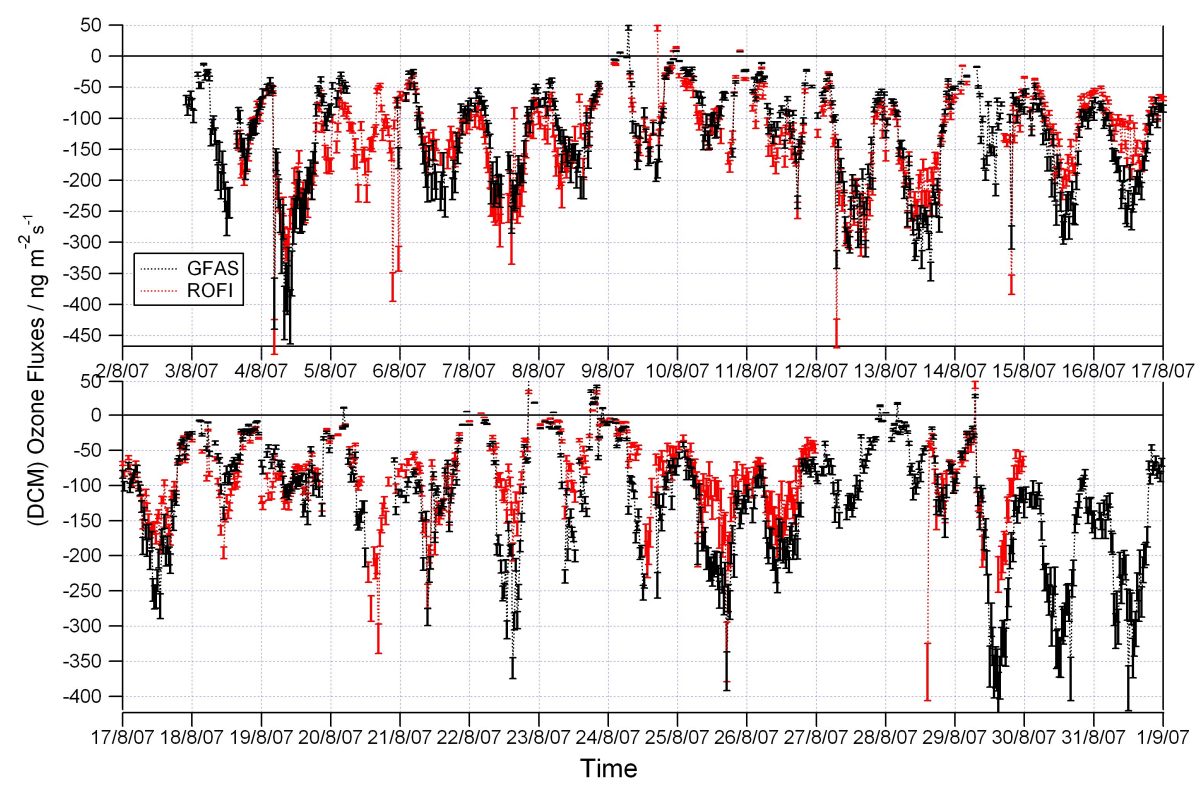

Fig. 4. Time series of fluxes by DCM for the GFAS and ROFI with error bars for the calculation method DCM. The top panel shows the time period from 2 August 2007 to 17 August 2007 and the bottom panel covers the period from 17 August 2007 to 1 September 2007.

To establish which calculation method is preferable, an empirical approach was used comparing the two analysers for each method and each disc period, checking which method provides the best match. Comparing the interquartile ranges (IQR) i.e. 50\% of the data, GFAS and ROFI fluxes by RM do not agree for disc periods 4, 6 and 7. The ROM fluxes give a good agreement for all periods. The DCM data also agree well for all periods. Comparing DCM with ROM, the quality of agreement of the IQR and median and mean flux values varies with the disc periods, but the DCM does slightly better overall. As DCM produces the closest agreement between the GFAS and ROFI for most of the time, DCM is used in the following section "Comparison of analysers".

The comparison shows that the choice of calculation method can be a substantial source of uncertainty in the absolute ozone fluxes. Exact data quality control and calibration procedures are often not described sufficiently when ozone fluxes from fast response dry chemiluminescence analysers are reported. If results are to be compared, a clear description of the calibration method is essential for interpretation. If these analysers are to be used in a network of flux sites, a tested protocol on how the ozone data are to be treated needs to be implemented to minimise the source of uncertainty in fluxes from the choice of calibration method.

\subsection{Comparison of analysers and uncertainties associated with the detection method}

The comparison of the GFAS and ROFI analysers is made using fluxes calculated by the Disc Calibration Method. Focussing on the performance of the two analysers compared to each other, Fig. 4 shows the time series of ozone fluxes for the GFAS and ROFI instruments.

There are some days when the two analysers agree well, e.g. on 7, 10, 12 and 21 August 2007. However there are also days when the ROFI measures smaller fluxes than the GFAS, as on 13, 15, 16, 17, 22, 25 and 26 August 2007. The measurements can differ up to about a factor of two. This is starkly illustrated by fluxes on 21 and 22 August in Fig. 5, where one day with very good agreement is followed by a day with poor agreement. The relationship of meteorological conditions, such as e.g. relative humidity, with flux differences by the analysers has been investigated and no consistent link across the whole dataset could be found (Fig. 6).

To understand the differences in fluxes that occurred for the some periods and not others, it is important to exclude a change in instrument performance as the reason for the observed difference. Figure 7 shows the frequency-weighted, "log-binned" power spectra (top panels) and co-spectra (bottom panels) for a selected time during 12, 17, 21 and $26 \mathrm{Au}-$ gust 2007. These are representative of periods when the analysers agree (12 and 21 August) and when they do not agree (17 and 26 August). It is evident that both analysers performed and compare relatively well for all the selected periods. The power spectra follow the $f^{-2 / 3}$ slope reasonably well as expected for turbulence dissipation within the inertial sublayer (Stull, 1988). For the period when fluxes do not agree the power in the GFAS is larger than in the ROFI (for $n=0.1$ to 1 ) which could be a reason for the observed differences in the flux. The increases of the spectra with a slope of $f^{+1}$ at frequencies above about $2 \mathrm{~Hz}$ indicate high white noise levels at those frequencies. In both cases the noise in the fast ozone signal is not correlated with the instantaneous 


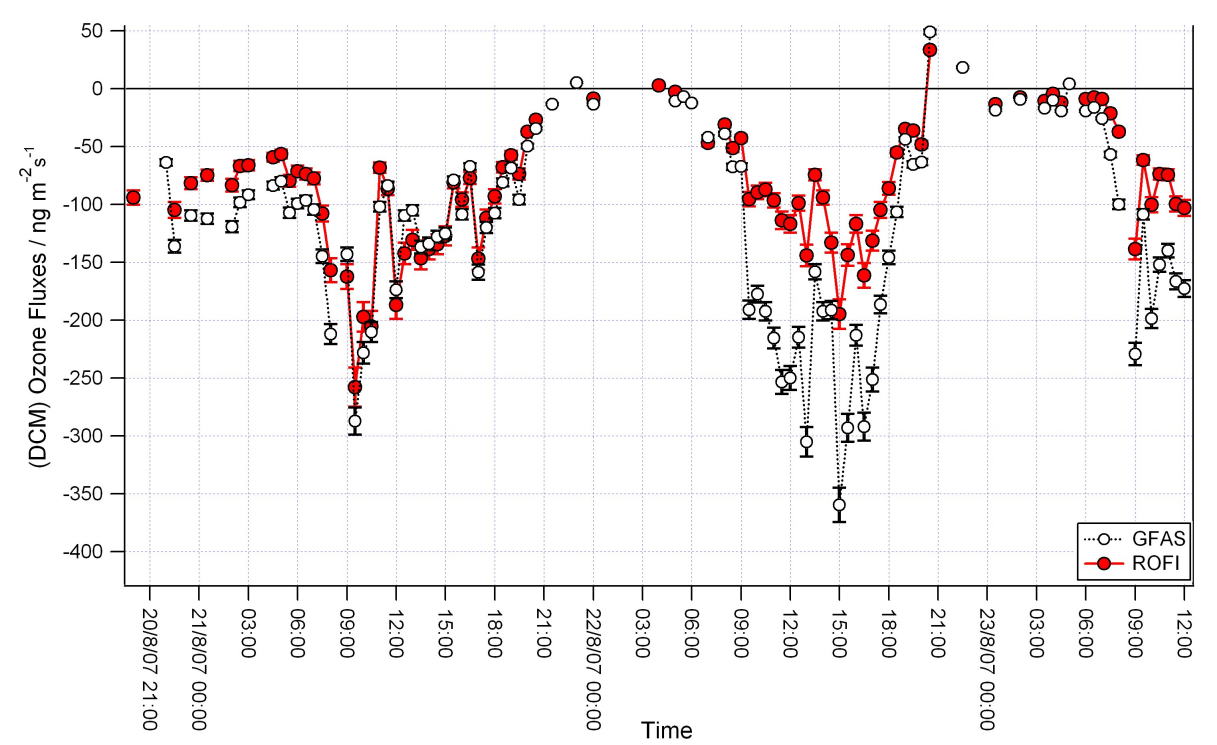

Fig. 5. GFAS and ROFI ozone fluxes with error bars for the calculation method DCM for 21 and 22 August 2007.

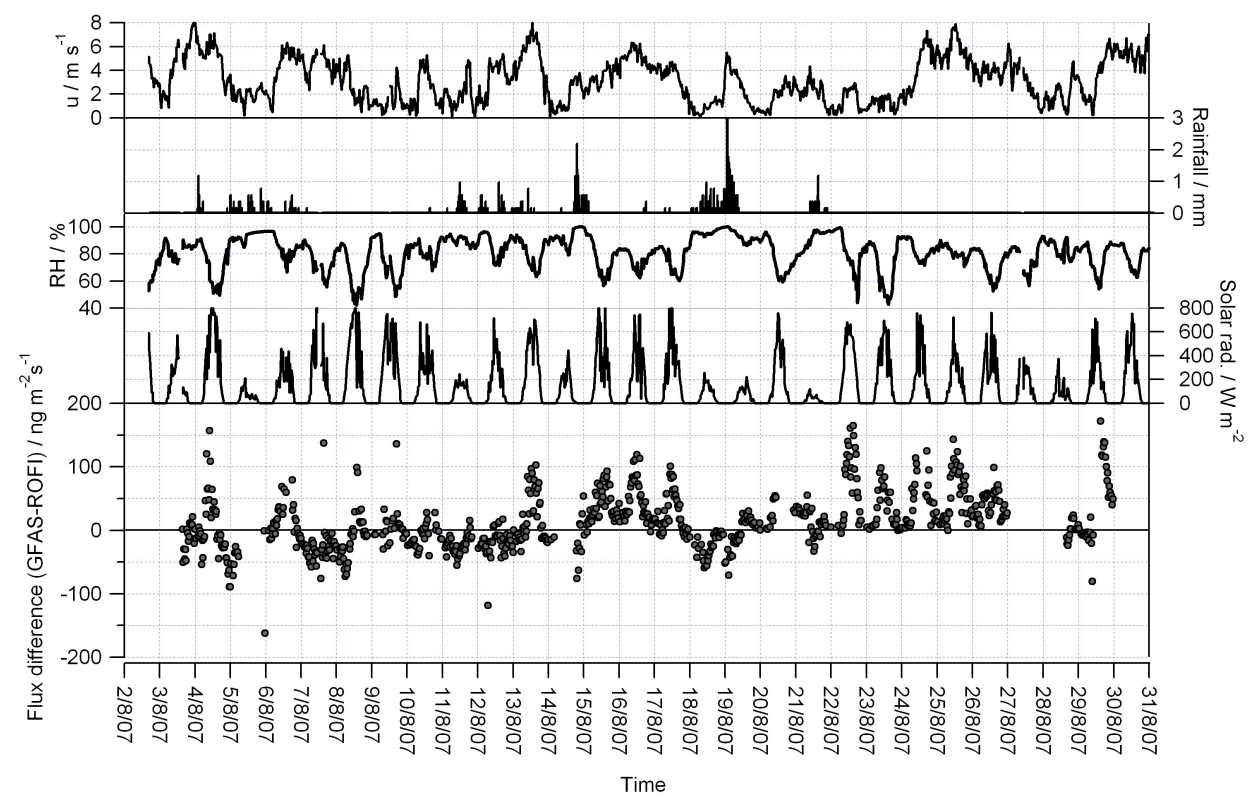

Fig. 6. Time series of wind speed, rainfall, relative humidity, solar radiation and difference of mean GFAS and ROFI fluxes by DCM.

vertical wind speed fluctuation $w^{\prime}$ as the co-spectra continue to follow the $f^{-4 / 3}$ slope at the higher frequencies to a large degree (Stull, 1988). Alternative ways of inspecting co-spectra such as non-weighted co-spectra or ogives (not shown here) do not reveal any further differences in shapes or patterns that could help explain the differences in the two analysers. It is also possible to compare cross-correlation products between the vertical windspeed and the fast ozone output. For the flux calculations, the cross-correlation between the vertical windspeed and the ozone signal is calculated for each flux-averaging period and the point at which maximum cross-correlation within a given time lag window occurs, determines the lag of the ozone signal compared to the instantaneous vertical windspeed. When comparing the cross-correlations (Fig. 8) for different periods, it is clear that the shape of the curves is the same for periods when the two analysers compare well (21 August 2007). For periods when fluxes differ, the cross-correlation differs slightly (17 August 2007). This indicates that some of the observed difference in flux may be a result of the way the lag time between the vertical windspeed and fast ozone signal is found. The time lag window was set to $2 \mathrm{~s}$ which is reasonable for 


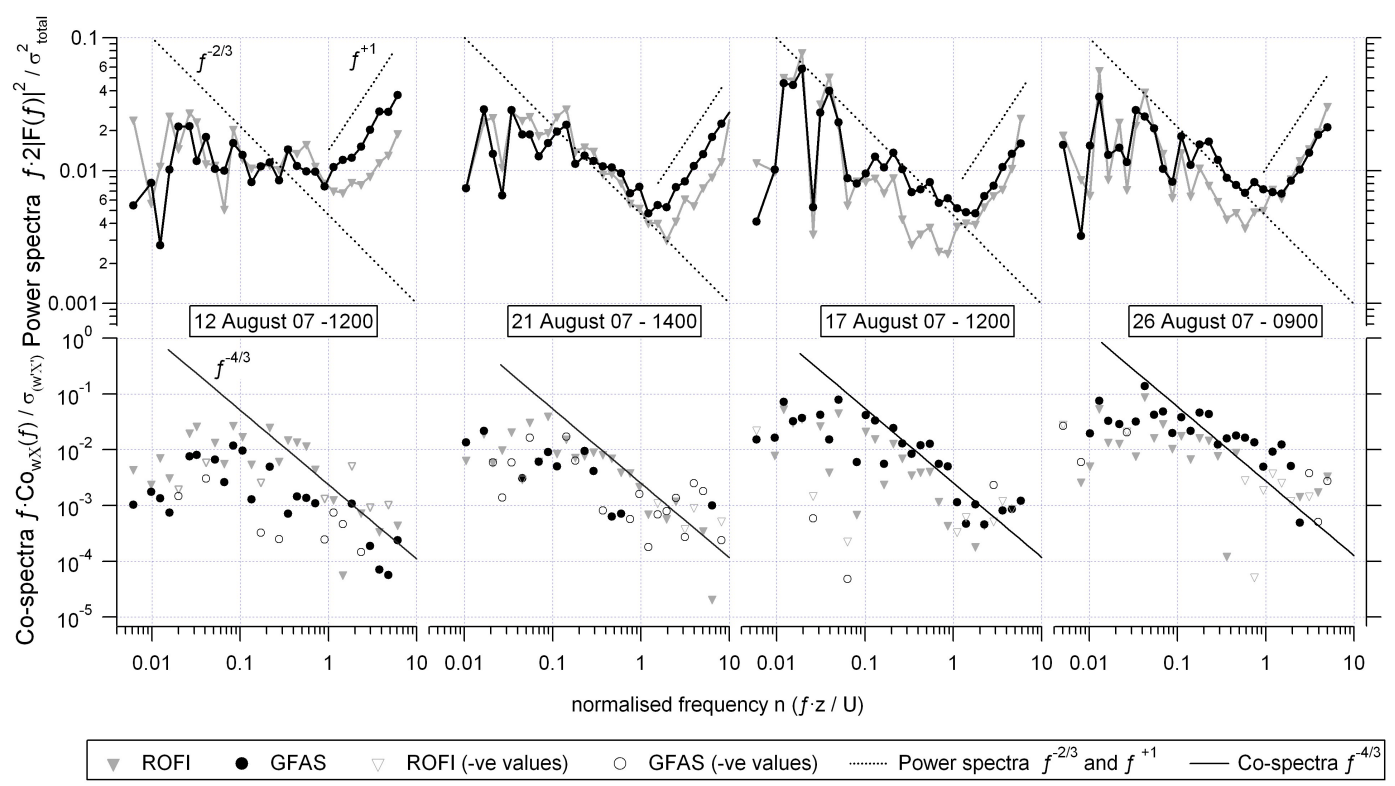

Fig. 7. Frequency weighted power spectra (top panels) for GFAS and ROFI analysers for the 12 and 21 August (when absolute fluxes agree) and 17 and 26 August (when absolute fluxes do not agree) and corresponding co-spectra (bottom panels) showing that high frequency noise in analyser $\left(f^{+1}\right.$ slope $)$ is not correlated with $w^{\prime}$ and co-spectra follow $f^{-4 / 3}$ slope.

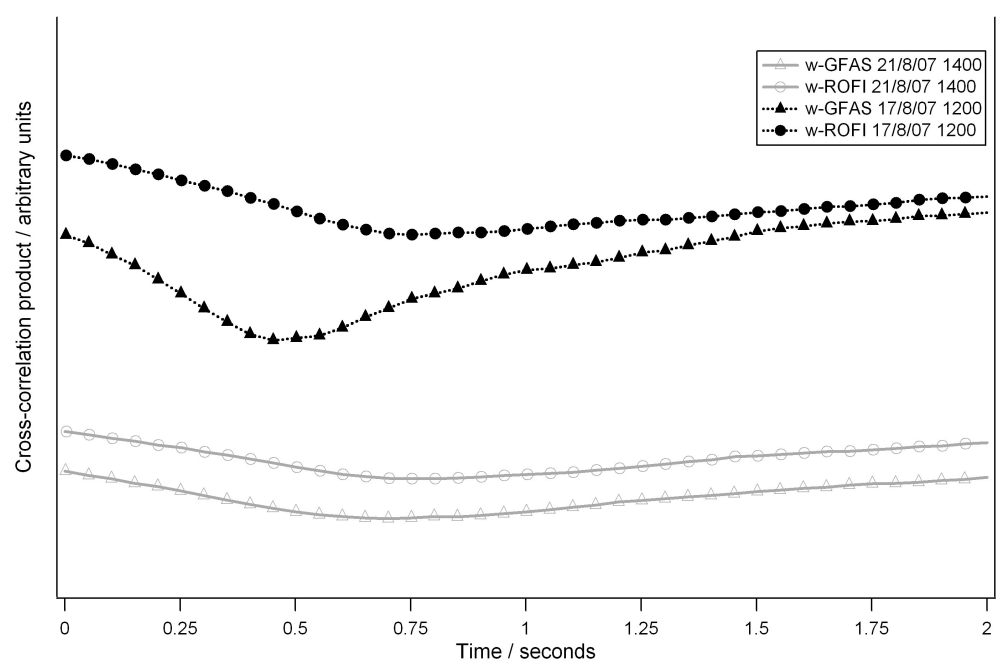

Fig. 8. Cross correlations of vertical windspeed $w$ with both GFAS and ROFI. Black lines with solid symbols are for periods when fluxes do not agree (17 August 2007) and grey lines with open symbols for periods when fluxes agree (21 August 2007).

the length of the inlet and flow rate. Also, when comparing lag times for a period when fluxes agree (e.g. whole day of 21 August 2007), lag times are variable for both GFAS and ROFI, indicating that using e.g. a fixed time lag, instead of one found by maximising the cross-correlation between $w^{\prime}$ and $X^{\prime}$, would not remove the difference between the GFAS and ROFI fluxes. The question whether variable fan performance is the reason for the difference in the cross-correlation function can not be answered definitively as flow rates or fan voltage was not measured during the campaign. The fans are designed to produce flow rates around $100 \mathrm{~L} \mathrm{~min}^{-1}$ and vari- ability of about $10 \%$ should not impact on the sensitivity and response time (Guesten et al., 1992). The mean lag times of the ROFI were generally larger than those of the GFAS (mean ROFI $0.75 \mathrm{~s}$ and mean GFAS $0.50 \mathrm{~s}$ ), and values close to the mean were found for all periods shown in Fig. 7, which implies that the analysers operated normally. The larger lag times for the ROFI can be explained by the slightly larger internal volume of the inlet tube. The length of both inlets is the same with $1.2 \mathrm{~m}$, but the inlet diameter of the ROFI inlet is larger by $0.007 \mathrm{~m}$. 


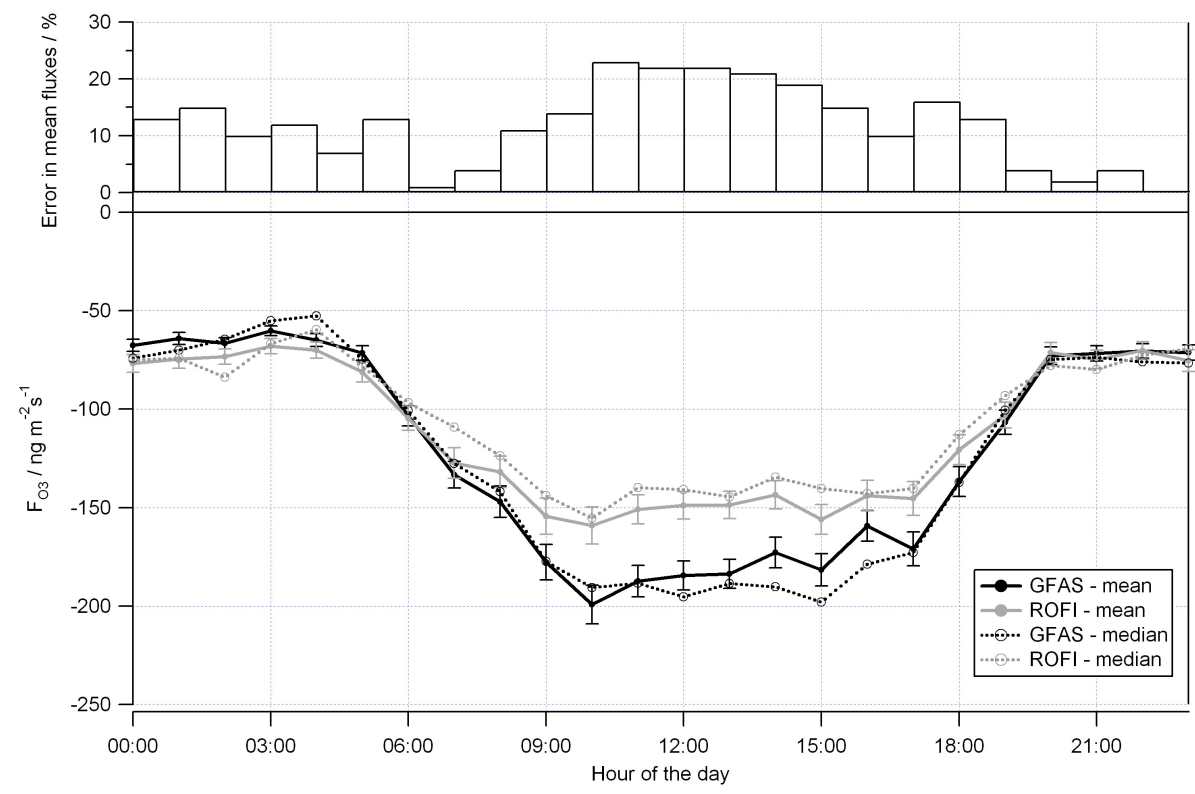

Fig. 9. Mean diurnal cycle of GFAS and ROFI fluxes with error bars from calculation method (bottom panel) and mean error based on the discrepancy between mean GFAS and mean ROFI fluxes (top panel).

It is generally assumed that all factors but the target discs remain the same for both analysers. The difference in crosscorrelation functions could potentially be due to the change in fan performance but it could also be a result of other factors: for instance there could be differences as a result of the different thermal properties of the analysers themselves or changes in the characteristics of the target discs are also possible.

If these ozone flux measurements had been made with only one of the analysers, each dataset would have been accepted as a good estimate of the flux. These measurements however show that, although absolute flux values might be reasonable in terms of expected ranges and the data have been deemed of sufficient quality by the adopted QC protocols, ozone fluxes still might potentially include a considerable uncertainty. Based on this study, the true half-hourly flux value might differ from the measurement by up to a factor of two (Fig. 5) under some circumstances.

The mean diurnal cycle in fluxes for both analysers shows that the fluxes on average agree during night time when fluxes are smaller, but larger discrepancies are evident during daytime, 9 am to $6 \mathrm{pm}$ (Fig. 9). This difference effectively constitutes the true error in the measurements and the mean error, as estimated from the mean GFAS and ROFI flux, can range from $0-23 \%$. The overall daily mean error is $12 \%$. The median diurnal cycles do not differ greatly from the mean cycles and differences in fluxes from the two instruments are equally evident in the median statistic. It might be argued based on the averaged diurnal profile that there must be a systematic difference between the two analysers, but it is important to consider that the absolute differences can change dramatically from one day to the next (Fig. 5). This highlights the variability and uncertainty that is associated with these analysers.

Three main sources of ozone flux uncertainty were quantified for the eddy covariance fast response dry chemiluminescence analysers used in this study:

(i) Uncertainty in the regression parameters is dependent on how well the fast ozone analyser's mean output correlates with the absolute ozone concentrations. Based on all disc periods, analysers and calculation methods, the $2 \sigma$ error in the mean flux value can range from $8-34 \%$. Overall for the whole dataset and both analysers and calculation methods, the mean $2 \sigma$ error in the mean flux is $16 \%$.

(ii) Uncertainty as result of the chosen method to calculate absolute ozone fluxes arises from the different approach to "calibrate" the relative analyser output. Comparing the Ratio Method (RM) and Ratio Offset Method (ROM) on a disc by disc basis, $50 \%$ of the time the two methods agreed within the $2 \sigma$ error associated with the regression parameters (see point i). For the remaining half of the time, the flux values differed on average by $13 \%$. Comparing the ROM and Disc Calibration Method (DCM) for each disc period, the methods agreed within error about $20 \%$ of the time. For the remaining time period, the flux values differed between the methods on average by $8 \%$.

(iii) Uncertainty as result of disc variability or analyser performance can cause the single most sizeable error. The analyser comparison for DCM revealed that there can be periods when the flux can be different by a factor of 2 . The mean diurnal cycle reveals that true mean error can be up to $23 \%$, with the largest errors on average occurring around 
midday. If measurements are made only with one analyser and no additional validation option is available, the error in the mean daily flux values from the variability in discs or analyser performance should be assumed to about $\pm 12 \%$.

Uncertainties from point (i) can potentially be minimised by using a stricter quality control threshold for the regressions, i.e. a higher regression coefficient $R^{2}$. This might result in a greater loss of data points that are not deemed of sufficient quality and there might be a trade off between final $\%$ data capture and \% error in the regression parameters. Uncertainties from (ii) are especially important to consider when ozone fluxes from different datasets are to be compared. Unless the exact procedure and calculation method is reported, differences in fluxes could be due to the choice of calculation method. It is recommended that a common data quality control and flux calculation method procedure is applied when these analysers are used in ozone flux networks. Minimising uncertainties from (iii) could be reduced by increasing the knowledge of what causes the variability. For instance, laboratory studies testing how the high frequency response varies with an increased accumulated ozone exposure and whether the relationship with the mean output remains linear could be useful. There are other open questions such as what the exact surface activation mechanism during the pre-ozonisation stage is, and the influence of a range of humidities and temperatures on the disc sensitivity with an increased accumulated ozone exposure.

\section{Conclusions}

Two dry chemiluminescence fast response ozone analysers have been run side by side for eddy covariance ozone flux measurements at a grassland site near Edinburgh, Scotland during August 2007. A detailed quality control procedure for the data obtained by the GFAS/ROFI type dry chemiluminescence ozone analysers has been produced. Uncertainties associated with this measurement method have been investigated. In this study, the same sonic anemometer, logging and analysis software was used for both fast response ozone analysers, so some sources of error could be eliminated or can be at least considered the same for the ozone fluxes obtained by the two instruments. One potential source of error arises from the way the lag time between the vertical velocity and fast ozone signals are determined. The underlying reason for this to be a source of error during some periods and not others could not be identified unambiguously. It is speculated that reported differences are likely to be due to the detection technique.

The GFAS/ROFI type dry chemiluminescence (DC) analysers have clear advantages over other fast response ozone instruments: they are simple in their operation, they have low power requirements and they are relatively lightweight, small and robust which makes them ideal for use in a variety of environments. The comparison of the two analysers in this study shows that on average the diurnal trend and fea- tures, but not the absolute values, of ozone deposition are captured well. On some days there is an excellent agreement between the analysers and confidence in the accuracy of the ozone flux can be gained from that agreement. However there are also days when the fluxes do not agree so well and the underlying cause of the difference could not be identified definitively. Whilst for instance changes in analyser performance could play a role in affecting sensitivity and response time, the chemiluminescence target discs and reaction mechanism could constitute a source of uncertainty. In this study, absolute ozone concentration measurements were made by a reliable and well calibrated UV absorption instrument and thus errors in the absolute ozone concentrations were assumed negligible. Regressions of the relative ozone output against the absolute concentrations show that the degree of scatter around the linear fitted line varies from disc to disc. Comparison of the absolute concentrations with those measured by the fast response analysers also shows that sensitivity does not only vary from disc to disc and decreases with time as the reagents are consumed by the reaction with ozone, but sensitivity can also vary at shorter timescales. It is therefore reasonable to focus on the disc as a source of uncertainty. The advantages of these analysers are numerous and some errors can clearly be minimised through the experimental set-up and by careful monitoring of disc sensitivity during the measurement period as well as rigorous data quality control at the data processing stage.

\section{Recommendations}

It is recommended to monitor the raw ozone output closely during ozone flux measurements and to replace the disc regularly, ideally before sensitivity noticeably decreases. To additionally provide confidence in the ozone fluxes, high quality ozone concentration measurements at sampling intervals at the order of tens of seconds to minutes would be useful to verify the stability of disc sensitivity at flux-averaging time scales. Another way to achieve greater confidence during field experiments is by running two analysers side by side. In addition to greater confidence in the final ozone fluxes, greater data coverage might also be achieved.

Acknowledgements. JBAM acknowledges the $\mathrm{PhD}$ studentship funding by the UK Natural Environment Research Council (NER/S/A/2006/14037) and additional CASE support by the NERC Centre for Ecology \& Hydrology. The infrastructure of the Easter Bush field site is supported by the European Integrated Projected NitroEurope. The authors thank James Dorsey and Robert Storeton-West for help with instrumentation before and during the campaign. The authors also thank the three anonymous reviewers for their constructive comments on the manuscript.

Edited by: O. Torres 


\section{References}

Ashmore, M. R.: Assessing the future global impacts of ozone on vegetation, Plant Cell Environ., 28, 949-964, 2005.

Bassin, S., Calanca, P., Weidinger, T., Gerosa, G., and Fuhrer, J.: Modeling seasonal ozone fluxes to grassland and wheat: model improvement, testing, and application, Atmos. Environ., 38, 2349-2359, 2004.

Bauer, M. R., Hultman, N. E., Panek, J. A., and Goldstein, A. H.: Ozone deposition to a ponderosa pine plantation in the Sierra Nevada Mountains (CA): A comparison of two different climatic years, J. Geophys. Res., 105(D17), 22123-22136, 2000.

Businger, J. A.: Evaluation of the accuracy with which dry deposition can be measured with current micrometeorological techniques, J. Clim. Appl. Meteorol., 25, 1100-1124, 1986.

Coyle, M.: The Gaseous Exchange of Ozone at Terrestrial Surfaces: Non-stomatal Deposition to Grassland, PhD thesis, University of Edinburgh, UK, 2005.

Derwent, R. G., Simmonds, P. G., Manning, A. J., Spain, T. G.: Trends over a 20-year period from 1987 to 2007 in surface ozone at the atmospheric research station, Mace Head, Ireland, Atmos. Environ. 41, 9091-9098, 2007.

Emberson, L. D., Wieser, G., and Ashmore, M. R.: Modelling of stomatal conductance and ozone flux of Norway spruce: comparison with field data, Environ. Pollut., 109, 393-402, 2000.

Finkelstein, P. L. and Sims, P. F.: Sampling error in eddy correlation flux measurements, J. Geophys. Res., 106(D4), 3503-3509, 2001.

Foken, T. and Wichura, B.: Tools for quality assessment of surface based flux measurements, Agr. Forest Meteorol., 78, 83$105,1996$.

Fuhrer, J.: Ozone risk for crops and pastures in present and future climates, Naturwissenschaften, 96, 173-194, doi:10.1007/s00114-008-0468-7, 2009.

Gallagher, M. W., Beswick, K. M., and Coe, H.: Ozone deposition to coastal waters, Q. J. Roy. Meteor. Soc., 127, 539-558, 2001.

Gruenhage, L., Haenel, H-D., and Jaeger, H-J.: The exchange of ozone between vegetation and atmosphere: micrometeorological measurement techniques and models, Environ. Pollut., 109, 373$392,2000$.

Guesten, H. and Heinrich, G.: On-line measurements of ozone surface fluxes: Part 1 Methodology and Instrumentation, Atmos. Environ., 30, 897-909, 1995.

Guesten, H., Heinrich, G., Schmidt, R. W. H., and Schurath, U.: A novel ozone sensor for direct eddy covariance measurements, J. Atmos. Chem., 14, 73-84, 1992.

Hodgeson, J. A., Krost, K. J., O'Keeffe, A. E., and Stevens, R. K.: Chemiluminescent measurement of atmospheric ozone: Response characteristics and operating variables, Anal. Chem., 42, 1796-1802, 1970.
Keronen, P., Reissell, A., Rannik, U., Pohja, T., Siivola, E., Hiltunen, V., Hari, P., Kulmala, M. and Vesala, T.: Ozone flux measurements over a Scots pine forest using eddy covariance method: performance evaluation and comparison with fluxprofile method, Boreal Environ. Res., 8, 425-443, 2003.

Lee, X., Massman, W., and Law, B.: Handbook of Micrometeorology: A Guide for Surface Flux Measurements and Analysis, Kluwer Academic Publisher, Dordrecht, 2004.

Mckendry, I. G, Steyn, D. G., O'Kane, S., Zawar-Reza, P., and Heuff, D.: Lower tropospheric ozone measurements by light aircraft equipped with chemiluminescent sonde, J. Atmos. Ocean. Tech., 15, 136-143, 1998.

Milford, C., Theobald, M. R., Nemitz, E., Sutton, M. A.: Dynamics of ammonia exchange in response to cutting and fertilising in an intensively-managed grassland, Water Soil Air Poll. Focus, 1, 167-176, 2001.

Pearson, R. Jr.: Measuring ambient ozone with high sensitivity and bandwidth, Rev. Sci. Instrum., 61, 907-916, 1990.

Rummel, U., Ammann, C., Kirkman, G. A., Moura, M. A. L., Foken, T., Andreae, M. O., and Meixner, F. X.: Seasonal variation of ozone deposition to a tropical rain forest in southwest Amazonia, Atmos. Chem. Phys., 7, 5415-5435, 2007, http://www.atmos-chem-phys.net/7/5415/2007/.

Schurath, U., Speuser, W., and Schmidt, R.: Principle and application of a fast sensor for atmospheric ozone, Fresen. J. Anal. Chem., 340, 544-547, 1991.

Speuser, W., Sahand, S., and Schurath, U.: A novel fast-response chemiluminescence sonde for routine soundings of stratospheric ozone up to $1.5 \mathrm{mbar}$, in: Ozone in the Atmosphere, edited by: Bojkov, R. D. and Fabian, P. A., Deepak Publishing, Hampton Virginia, USA, 747-750, 1989.

Stull, R. B.: An Introduction to Boundary Layer Meteorology, Kluwer Academic Publishers, Dordrecht, the Netherlands, 666 pp., 1988.

Tuovinen, J-P., Ashmore, M. R., Emberson, L. D., and Simpson, D.: Testing and improving the EMEP ozone deposition module, Atmos. Environ., 38, 2373-2385, 2004.

Tuovinen, J-P., Aurela, M., and Laurila, T.: Resistances to ozone deposition to a flark fen in the northern aapa mire zone, J. Geophys. Res., 103(D14), 16953-16966, 1998.

Weather: Weather Log, 62 (Eq. 10), available at: www.interscience. wiley.com/weather, 2007.

Weinheimer, A. J.: Chemical Methods: Chemiluminescence, Chemical Amplification, Electrochemistry and Derivatization, in: Analytical Techniques for Atmospheric Measurement, edited by: Heard, D. E., Blackwell Publishing Ltd, Oxford, 311-360, 2006.

Wilczak, J. M., Oncley, S. P., and Stage, S. A.: Sonic anemometer tilt correction algorithms, Bound-Lay. Meteorol., 99, 127-150, 2001.

Wohlfahrt, G., Hoertnagel, L., Hammerle, A., Graus, M., and Hansel, A.: Measuring eddy covariance fluxes of ozone with a slow-response analyser, Atmos. Environ., 43, 4579-4576, 2009. 